Vol. 01, No. 04, octubre - diciembre 2021, 43-55

\title{
Cultivo dual in vitro de cepas nativas de Trichoderma spp. frente a Botrytis sp. patógeno de Passiflora ligularis Juss
}

\section{Dual in vitro culture of native strains of Trichoderma spp. against Botrytis sp. pathogen of Passiflora ligularis Juss}

\author{
Luis T. Acosta ${ }^{1, \mathrm{a}, *}$, Deysi K. Azania ${ }^{1, \mathrm{~b}}$, Rolando Azania ${ }^{1, \mathrm{c}}$
}

\footnotetext{
${ }^{1}$ Universidad Nacional Daniel Alcides Carrión, Oxapampa, Perú.

a Ing., \ luistibhy@hotmail.com, iD https://orcid.org/0000-0002-1276-1777

b Ing., $\$ deysikaf@hotmail.com, (iD https://orcid.org/0000-0003-1441-7897

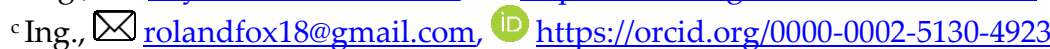

* Autor de Correspondencia: Tel. +51941696300

http://dx.doi.org/10.25127/riagrop.20214.720

http://revistas.untrm.edu.pe/index.php/RIAGROP

revista.riagrop@untrm.edu.pe

Recepción: 11 de agosto 2021

Aprobación: 28 de agosto 2021

Este trabajo tiene licencia de Creative Commons. Attribution-NonCommercial-ShareAlike $\quad 4.0$ International Public License - CC-BY-NC-SA 4.0

\section{Resumen}

El objetivo de la investigación fue evaluar el nivel de antagonismo de trece cepas nativas de Trichoderma spp. frente a Botrytis sp. A nivel in vitro, se evaluó mediante el método de cultivo dual en placas Petri con Papa Dextrosa Agar (PDA), donde se observó crecimiento radial, competencia por espacio y nutrientes, micoparasitismo y grado de inhibición; se utilizó el diseño completamente aleatorio, con 27 tratamientos y 4 repeticiones. Los resultados del enfrentamiento de las cepas de Trichoderma spp. frente a Botrytis sp. mostraron un superior crecimiento radial para Trichoderma. Por otro lado, todos los tratamientos de Trichoderma spp. lograron restringir el desarrollo de Botrytis sp., con respecto a su testigo. En la competencia por espacio y nutrientes, las cepas nativas evaluadas de Trichoderma pueden ser un recurso valioso para su evaluación en el control biológico de otras enfermedades en el cultivo de granadilla, como también en el establecimiento de dosis efectivas de las cepas de Trichoderma spp. para el control preventivo de plántulas de granadilla a nivel de invernadero y campo. 
Palabras claves: granadilla, control biológico, enfermedades, dosis.

\begin{abstract}
The objective of the research was evaluate the level of antagonism of thirteen native strains of Trichoderma spp. against Botrytis sp. At the in vitro level, it was evaluated by the method dual culture in Petri dishes with Potato Dextrose Agar (PDA), where growth was observed radial, space competition and nutrients, mycoparasitism and degree inhibition; the design was used completely random, with 27 treatments and 4 repetitions. The results of the confrontation of the strains of Trichoderma spp. versus Botrytis sp. showed a superior radial growth for Trichoderma. On the other hand, all Trichoderma spp. treatments managed to restrict the development of Botrytis sp. regarding your witness; in the competition for space and nutrients. The native strains evaluated from Trichoderma can be a valuable resource for evaluation in the biological control of other diseases in the cultivation of sweet granadilla, as well as in the establishment of effective doses of the strains of Trichoderma spp. for the preventive control of sweet granadilla seedlings at the greenhouse and field level.
\end{abstract}

Keywords: sweet granadilla, biological control, diseases, dose.

\section{INTRODUCCIÓN}

En el antagonismo, se conoce un grupo importante de hongos y bacterias que presentan efecto antagónico sobre otros microorganismos. Este efecto es aprovechado por el hombre para la regulación, tanto de patógenos cuyo hábitat es el suelo, como aquellos que se desarrollan en la parte foliar de las plantas (Infante et al. 2009). Los antagonistas contribuyen a la atenuación de los daños que causan las enfermedades en los agroecosistemas donde existan condiciones para su desarrollo y conservación. Entre las especies más ampliamente estudiadas $\mathrm{y}$ aplicadas al control biológico, se encuentran las del género Trichoderma, debido a su eficaz control, capacidad reproductiva, plasticidad ecológica y efecto estimulante sobre los cultivos (Infante et al. 2009).

La producción de granadilla (Passiflora ligularis Juss.) en Oxapampa es afectada por enfermedades, sobre todo, en los meses de mayor precipitación, causada por Botrytis sp., Fusarium spp. y Colletotrichum spp. entre otros.
El control de estos hongos patógenos, actualmente, se realiza con plaguicidas que son aplicados al follaje, a las semillas y al suelo. Su uso origina problemas tales como la destrucción de la capa de ozono; contaminación del ambiente, al acumularse en el agua, suelo, plantas y animales; peligro para la salud de los agricultores cuando el uso es sin ningún tipo de protección y precaución; enfermedades en humanos y animales cuando se consumen productos vegetales contaminados con residuos tóxicos que pueden producir la muerte; exterminio de microorganismos benéficos, muchas veces alteran el equilibrio ecológico; resistencia por parte de los patógenos y conversión en plaga de aquellos organismos no considerados como tales.

La búsqueda de alternativas al uso de los plaguicidas direcciona la mirada de los investigadores hacia los microorganismos, para su utilización como agentes de control biológico, en el control de patógenos de plantas. Esto es por la diversidad de microorganismos 
nativos factibles de utilizarse, tanto hongos como bacterias, reconocidos como supresores de patógenos de plantas, limitando la severidad de las enfermedades en los cultivos (Memenza, 2009).

Trichoderma spp. es de distribución cosmopolita. Se presenta principalmente en zonas que contienen materia orgánica o desechos vegetales en descomposición. Los mecanismos de acción que presentan distintas especies del género Trichoderma se apoyan principalmente en la fuerte capacidad competitiva por espacio y nutrientes, también se han reportado como parásitos de gran número de organismos fungosos fitopatógenos (Esparza, 2009). En el estudio de investigación aislamiento, identificación y caracterización cultural de cepas nativas de Trichoderma spp. en la provincia de Oxapampa, se caracterizó culturalmente en medio de cultivo PDA 15 cepas nativas de Trichoderma y se describió las características macroscópicas y microscópicas más importantes, siendo las especies $T$. viride $\mathrm{y}$ T. harzianum las más abundantes de esta colección (Rosales 2012). Por tanto, es necesario la prueba in vitro de trece cepas nativas de Trichoderma spp. frente a Botrytis sp., considerado como uno de los principales patógenos de P. ligularis Juss. El objetivo de este trabajo fue evaluar el nivel de antagonismo de trece cepas nativas de Trichoderma spp. frente a Botrytis sp., resultados que permitirá a los investigadores disponer de información importante para la selección y uso de este controlador biológico.

\section{MATERIALES Y MÉTODOS}

\subsection{Lugar de estudio}

El experimento fue realizado en el Laboratorio de Fitopatología de la Universidad Nacional
Daniel Alcides Carrión, sede Oxapampa, ubicada en el distrito y provincia de Oxapampa, región de Pasco; las condiciones del ambiente dentro del laboratorio fueron en promedio una temperatura de $19{ }^{\circ} \mathrm{C}$ y humedad relativa de $67 \%$ durante la experimentación.

\subsection{Material biológico}

Aislamientos del hongo: Botrytis sp. patógeno de P. ligularis Juss. (granadilla) y las cepas nativas de Trichoderma spp. del cepario del Laboratorio de Fitopatología de la Universidad Nacional Daniel Alcides Carrión, sede Oxapampa.

\subsection{Medio de cultivo}

El medio de cultivo utilizado para el desarrollo y enfrentamiento in vitro de Botrytis sp. y cepas de Trichoderma spp. fue papa dextrosa agar (PDA).

\subsection{Metodología}

2.4.1. Purificación de cepas nativas de Trichoderma spp. a partir de cepario

Del cepario de conservación, se purificaron cada cepa de Trichoderma spp., de la siguiente manera: de un tubo de ensayo con Trichoderma spp., se extrajo una porción de micelio con un estilete (desinfectado por flameado) para trasladarlo a una placa Petri con medio de cultivo (PDA más cloranfenicol al $0.05 \%$ ); seguido se dejó incubar de tres a cinco días hasta observar crecimiento de micelio blanquecino; luego, de este micelio se extrajo las primeras hifas y se realizó un subcultivo que se dejó desarrollar hasta la esporulación (se observó micelio de color verduzco, característico de Trichoderma), este se utilizó como inóculo para el cultivo monoespórico y posterior conservación en el cepario. 


\subsubsection{Recolección de muestras del patógeno} (Botrytis sp.)

La colecta de frutos de granadilla (P. ligularis) con signos de Botrytis sp. se realizó en el sector de Navarra, entre las quebradas Tres Marías y Negro del distrito de Huancabamba, provincia de Oxapampa.

\subsubsection{Aislamiento del patógeno}

Botrytis sp.- El material muestra (fruto de granadilla) se aisló a partir del signo típico de Botrytis, que corresponde a una esporulación grisácea, previa observación de conidios con un estereoscopio. Seguido, se realizaron cortes de tejido en la zona de avance del patógeno de aproximadamente $17 \mathrm{~mm}$ de diámetro. Se desinfectó el material (tejido vegetal infectado) mediante inmersiones secuenciales con lejía Clorox (hipoclorito de sodio al $4 \%$ de concentración) diluida (1 $\mathrm{ml}$ por $1 \mathrm{Lt}$ de agua) por 30 segundos y finalmente se lavó en agua destilada estéril por 5 minutos. Luego tejido vegetal infectado, se sembró en placas Petri con PDA y se incubaron a $22 \pm 1{ }^{\circ} \mathrm{C}$ por dos días. A partir de las primeras hifas del hongo presentadas en el medio, se realizaron subcultivos para evitar posible contaminación.

\subsubsection{Identificación del patógeno}

Botrytis sp.- Se sembró discos de PDA con micelio de la cepa aislada en una placa Petri con medio de cultivo y se dejó desarrollar para luego observar al microscopio estructuras características de Botrytis sp., las que se verificaron con las claves de Barnett y Hunter (1998).

\subsubsection{Estudio de variables}

Crecimiento radial: se midió el avance del micelio del hongo a partir del segundo día después de la siembra, luego cada $24 \mathrm{~h}$. Competencia por área y nutrientes: se obtuvo con los radios de crecimiento radial para Trichoderma, Botrytis y testigos. Micoparasitismo: la capacidad antagónica de las cepas de Trichoderma spp. se determinó por la escala propuesta por Bell et al. (1982), detallada en tabla 1. Además, se evaluó la interacción de las hifas por observación con microscopio binocular Helmut Hund $\mathrm{GmbH}$ con aumento de 100X. Grado de inhibición (GI): se estimó con la fórmula utilizada por Sarro et al. (2010): $G I=\left[A_{p}-A_{p+a}\right] / A_{p} \times 100$, donde $A_{p}$ es el crecimiento radial del patógeno testigo y $A_{p+a}$ es el crecimiento radial del patógeno más el antagonista (cuando es enfrentado).

Tabla 1. Escala de estimación de micoparasitismo

\begin{tabular}{ccl}
\hline Clases & $\begin{array}{c}\text { Valor } \\
\text { (\%) }\end{array}$ & \multicolumn{1}{c}{ Descripción } \\
\hline 1 & 100 & $\begin{array}{l}\text { Trichoderma sobrecrece } \\
\text { completamente al patógeno y cubre } \\
\text { totalmente la superficie del medio. }\end{array}$ \\
2 & 92.05 & $\begin{array}{l}\text { Trichoderma sobrecrece las dos } \\
\text { terceras partes de la superficie del } \\
\text { medio. }\end{array}$ \\
3 & 50 & $\begin{array}{l}\text { Trichoderma y el patógeno crecen } \\
\text { aproximadamente la mitad de la } \\
\text { superficie y ninguno domina al otro. }\end{array}$ \\
4 & 7.95 & $\begin{array}{l}\text { El patógeno coloniza las dos terceras } \\
\text { partes de la superficie del medio y } \\
\text { resiste la invasión por Trichoderma. } \\
\text { El patógeno sobrecrece completamente } \\
\text { a Trichoderma y ocupa la superficie } \\
\text { total del medio. }\end{array}$ \\
\hline
\end{tabular}

\subsection{Análisis de datos}

En el experimento se usó el diseño completamente aleatorizado con veintisiete tratamientos y cuatro repeticiones. La unidad experimental consto de cuatro placas Petri con medio PDA a $\mathrm{pH}$ 6.5.

Los datos obtenidos de las diferentes variables se ordenaron en hojas de cálculo de Microsoft Excel y se procesaron con el software estadístico InfoStat, versión 2013; aplicando el análisis de 
varianza para determinar la significancia entre tratamientos y la prueba de significación de Duncan con $\alpha=0.05$ para establecer jerarquía e igualdad de los tratamientos.

\section{RESULTADOS Y DISCUSIÓN}

\subsection{Caracterización cultural e identificación de} Botrytis sp.

\subsubsection{Caracterización cultural}

La caracterización cultural de la cepa de código MANB01 (inóculo puro del hongo) en PDA, presento una tasa de crecimiento de micelio de $1.18 \mathrm{~cm} /$ día. Además, presento las siguientes características en placa con medio PDA: i) Aspecto de colonia: crecimiento normal, ni muy elevado ni muy sumergido, compacta, con una zona de esporulación al 4 día después de la siembra (dds) o incubación, las cuales se secan rápidamente; ii) Conidiación: compacta, blanca al 3 dds y tornándose marrón claro al 4 dds, dispuesta en un aro de conidiación de color marrón oscuro en forma de anillo de un grosor de $1 \mathrm{~cm}$, las zonas libres de conidiación son cubiertas por abundante micelio aéreo, blanquecino; iii) Color del reverso de la placa: incoloro no hay cambio en el medio de cultivo y iv) Olor de la colonia: sin olor.

\subsubsection{Identificación}

Se identificó morfológicamente la cepa Botrytis sp. en base a su estructura fructificante, características macroscópicas y microscópicas (figura 1) en contraste a las claves de Barnett y Hunter (1998) utilizadas; esta cepa se codifico como MANB01. Continuadamente se describen las características microscópicas: i) Conidióforos: de color marrón, erguidos, septados y ramificados, ramas pequeñas unidas en su punto de origen; ii) Conidios: ovalados en forma de huevo, presenta comúnmente un punto de unión sobresaliente ligeramente, de color marrón pálido y de tamaño: largo (promedio $10.2 \mu \mathrm{m}$ ) y ancho promedio (6.12 $\mu \mathrm{m})$ y iii) Esclerocios: presentes y poco abundantes, a los $15 \mathrm{dds}$.

Los caracteres microscópicos distintivos como: conidióforo, conidio, esclerocio y tipo de micelio concuerdan con las claves descritas por Barnett y Hunter (1998). Asimismo, hay cierta similitud con lo descrito por Pitt y Hocking (2009) quienes caracterizaron a B. cinérea en medios de cultivos CYA y MEA: el hongo mostro un micelio aéreo y/o aglomerado (flocoso), ritmo de crecimiento variado y el color del micelio fue de blanco a gris oscuro (producto de la conidiogénesis); también, observaron talos de duración indeterminada, presencia de conidióforos, conidios elipsoidales de 8 - $12 \mu \mathrm{m}$ de largo de pared lisa.

\subsection{Trichoderma spp. Frente a Botrytis sp.}

El crecimiento radial de micelio de Trichoderma spp. por efecto del enfrentamiento contra Botrytis sp. y sus testigos demostraron que existen diferencias estadísticas significativas ( $p$ $<0.05$ ), con un coeficiente de variabilidad de $6.5 \%$.

La prueba de comparación múltiple de Duncan ( $\alpha=0.05$ de probabilidad) jerarquizo las medias de los tratamientos estudiados (tabla 2) para el crecimiento radial de micelio de las cepas de Trichoderma en enfrentamiento y testigo. Resaltando estadísticamente a $T$. koningii (MHSJ11) con $4.47 \mathrm{~cm}$ y enfrentado con Botrytis sp. obtuvo $4.38 \mathrm{~cm}$; T. inhamatum (MOBSHO01) con $4.42 \mathrm{~cm}$ y enfrentado con Botrytis sp. logró $4.26 \mathrm{~cm}$; T. harzianum (MHMLL04) con $4.25 \mathrm{~cm}$ y T. atroviride (MHSJ01) enfrentado con Botrytis 
sp. consiguió $4.12 \mathrm{~cm}$ respectivamente. Posiblemente por una mayor velocidad de crecimiento y un rápido reconocimiento del patógeno. Por el contrario, la cepa $T$. longibrachiatum (MJHU01) con $1.3 \mathrm{~cm}, \mathrm{y}$ enfrentado con Botrytis sp., alcanzó $1.15 \mathrm{~cm}$ presentó un crecimiento radial significativamente inferior; tal vez por su crecimiento lento y nula diseminación en el medio que es una característica genética, acciones que no bloquean el desarrollo del patógeno. Además, cepas testigos y enfrentados de la misma especie no presentaron un crecimiento radial de micelio estadísticamente igual, como es el caso de las cepas T. harzianum (MHMLL04) con $4.25 \mathrm{~cm}$ y enfrentado con Botrytis sp. adquirió $4.09 \mathrm{~cm}$, seguido de $T$. atroviride (MHSJ01) - Botrytis sp. obtuvo $4.12 \mathrm{~cm}$ y T. atroviride (MHSJ01) adquirió $4.06 \mathrm{~cm}$ la cepa
T. citrinoviride (MHMLL03) logró $3.91 \mathrm{~cm} \mathrm{y}$ enfrentado con Botrytis sp. consiguió $3.46 \mathrm{~cm}$, precedido de la cepa T. atroviride (MHMLL06) con $3.79 \mathrm{~cm}$ y enfrentado con Botrytis sp. obtuvo $3.49 \mathrm{~cm}$ y finalmente por la cepa $T$. inhamatum (MOBSHO03) con $3.52 \mathrm{~cm}$ y enfrentado con Botrytis sp. alcanzó $3.02 \mathrm{~cm}$. Los resultados anteriores probablemente se deban a que hay cepas de una misma especie con características genéticas diferentes. En efecto, Trichoderma presenta actividad inhibitoria sobre diversos fitopatógenos (Dennis y Webster 1971). Asimismo, los resultados obtenidos en este estudio son similares a los de Chambers y Scott (1995), quienes utilizaron la misma técnica y encontraron que T. hamatum y T. pseudokoningii inhibieron el crecimiento micelial de Phytophthora spp. a nivel in vitro.

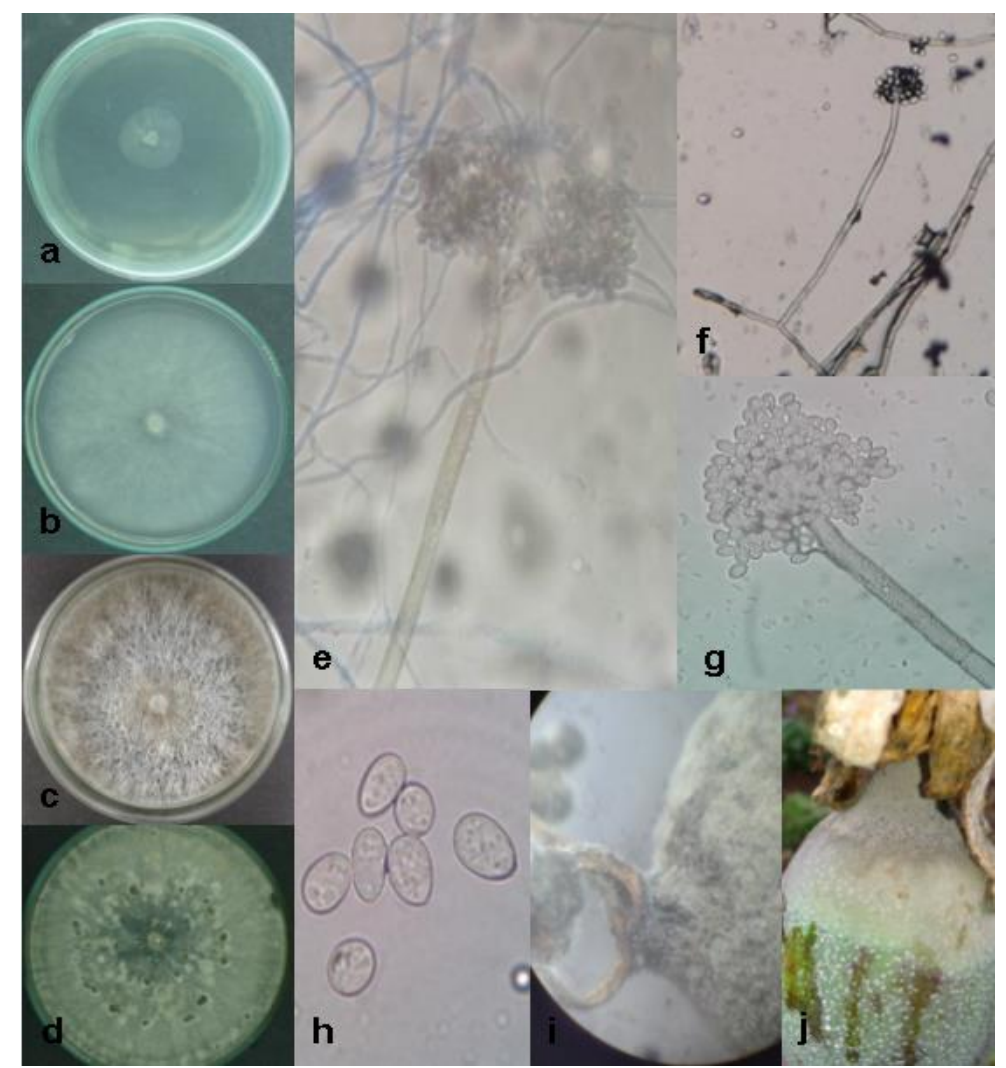

Figura 1. Características macroscópicas y microscópicas de Botrytis sp. (MANB01). Crecimiento del hongo en PDA: a. 1 dds, b. 3 dds, c. 8 dds, d. 15 dds (micelio con esclerocios), e. Conidióforo con conidios 40X, f. Conidióforo y conidios 10X, g. Conidióforo y conidios 40X, h. Conidios ovalados 100X, i. Conidiación (visto a estereoscopio 10X), j. Muestra: fruto de granadilla (P. Ligularis) con signos de Botrytis sp. 
Tabla 2. Comparacion de medias para el crecimiento radial de micelio de Trichoderma spp. por efecto del enfrentamiento con Botrytis sp. y testigos

\begin{tabular}{|c|c|c|}
\hline Trichoderma spp. vs Botrytis sp. y testigos & $\begin{array}{l}\text { Crecimiento radial de } \\
\text { micelio }(\mathrm{cm}) 3 \mathrm{dds} \text {. }\end{array}$ & $\begin{array}{c}\begin{array}{c}\text { Prueba de Duncan } \\
(\alpha=0.05)\end{array} \\
\end{array}$ \\
\hline T. koningii (MHSJ11) & 4.47 & a \\
\hline T. inhamatum (MOBSHO01) & 4.42 & $a b$ \\
\hline T. koningii (MHSJ11) vs Botrytis sp. & 4.38 & $a b$ \\
\hline T. inhamatum (MOBSHO01) vs Botrytis sp. & 4.26 & $a b c$ \\
\hline T. harzianum (MHMLL04) & 4.25 & $a b c$ \\
\hline T. atroviride (MHSJ01) vs Botrytis sp. & 4.12 & abcd \\
\hline T. harzianum (MHMLL04) vs Botrytis sp. & 4.09 & bcd \\
\hline T. atroviride (MHSJ01) & 4.06 & bcde \\
\hline T. koningii (MCHT11) vs Botrytis sp. & 4.05 & bcde \\
\hline T. koningii (MCHT11) & 3.98 & cdef \\
\hline T. citrinoviride (MHMLL03) & 3.91 & cdefg \\
\hline T. atroviride (MHMLL06) & 3.79 & defgh \\
\hline T. harzianum (MCHT07) & 3.71 & efgh \\
\hline T. harzianum (MOSA05) & 3.63 & fgh \\
\hline T. harzianum (MCHT07) vs Botrytis sp. & 3.62 & fgh \\
\hline Botrytis sp. & 3.55 & ghi \\
\hline T. atroviride (MOBSHO03) & 3.52 & hi \\
\hline T. harzianum (MOSA05) vs Botrytis sp. & 3.51 & hi \\
\hline T. atroviride (MHMLL06) vs Botrytis sp. & 3.49 & hi \\
\hline T. citrinoviride (MHMLL03) vs Botrytis sp. & 3.46 & hij \\
\hline T. strictipile (MCHT04) & 3.23 & ijk \\
\hline T. strictipile (MCHPSO3) & 3.23 & ijk \\
\hline T. strictipile (MCHT04) vs Botrytis sp. & 3.11 & $\mathrm{jk}$ \\
\hline T. strictipile (MCHPS03) vs Botrytis sp. & 3.03 & $\mathrm{k}$ \\
\hline T. atroviride (MOBSHO03) vs Botrytis sp. & 3.02 & $\mathrm{k}$ \\
\hline T. longibrachiatum (MJHU01) & 1.30 & 1 \\
\hline T. longibrachiatum (MJHU01) vs Botrytis sp. & 1.15 & 1 \\
\hline
\end{tabular}

El análisis de varianza para el crecimiento radial de micelio de Botrytis sp., por efecto del enfrentamiento contra cepas de Trichoderma spp. y testigo, indico la existencia de diferencias estadísticas altamente significativas ( $\mathrm{p}<0.01)$, con un coeficiente de variabilidad de $2.7 \%$.

La comparación múltiple de medias de Duncan ( $\alpha=0.05$ de probabilidad) presento un menor crecimiento radial de micelio $(2.41 \mathrm{~cm})$ para Botrytis sp. producto del enfrentamiento a $T$. atroviride (MHSJ01); evidenciando que la cepa $T$. atroviride (MHSJ01) es estadísticamente superior al resto (tabla 3), por inhibir el crecimiento micelial del patógeno; estos resultados posiblemente puedan deberse a la segregación de metabolitos secundarios y antibióticos que limitan el desarrollo normal de Botrytis. Por el contrario, la cepa T. harzianum (MCHT07) presento una menor inhibición en el crecimiento mecelial del patógeno $(3.96 \mathrm{~cm})$. Además, cepas de Trichoderma identificadas como una misma especie presentaron contingencia en el crecimiento del micelio de Botrytis con respecto al testigo; pero no presentaron un comportamiento similar, como se detalla: Botrytis sp. - $T$. atroviride (MOBSHO03) obtuvo $3.17 \mathrm{~cm}$ y Botrytis sp. - T. 
atroviride (MHMLL06) logró $3.39 \mathrm{~cm}$. De igual manera, Botrytis sp. - T. strictipile (MCHT04) alcanzó $3.22 \mathrm{~cm}$ y Botrytis sp. - T. strictipile (MCHPS03) obtuvo $3.44 \mathrm{~cm}$ y también Botrytis sp. - T. harzianum (MHMLL04) adquirió $3.39 \mathrm{~cm}$ y Botrytis sp. - T. harzianum (MCHT07) agenció $3.69 \mathrm{~cm}$. Los resultados anteriores probablemente puedan deberse a las diferentes características genéticas de cada cepa, en el rápido o lento desarrollo. Por otro lado, las cepas de Trichoerma enfrentadas al patogeno: Botrytis sp. - T. koningii (MHSJ11) con $3.43 \mathrm{~cm} \mathrm{y}$ Botrytis sp. - T. koningii (MCHT11) con $3.46 \mathrm{~cm}$ fueron las que presentaron efecto antagónico estadísticamente igual en el crecimiento radial de micelio de Botrytis, posiblemente se deba a que presentan las mismas características de reconocimiento y reacción frente al patógeno. El crecimiento radial de micelio de Botrytis sp. fue variado con respecto a su testigo, producto de la interacción antagónica con las cepas de Trichoderma spp.; asimismo, hay variación en la efectividad de las diferentes cepas de Trichoderma. Asimismo, Campbell (1989) ha observado que las cepas de Trichoderma spp. varían en su efecto antagónico debido a la versatilidad de mecanismos que posee, y que estos se expresan según las características genéticas prevalentes. Cabe agregar que, el sobrecrecimiento del micelio de Trichoderma en el micelio de un patógeno, es una característica ventajosa en la disputa de colonizar un área; esta característica reduce o detiene completamente el desarrollo del micelio del patógeno (Dennis y Webster 1971).

Tabla 3. Promedio de crecimiento radial de micelio de Botrytis sp. por efecto del enfrentamiento contra Trichoderma spp. y testigo

\begin{tabular}{lcc}
\hline \multicolumn{1}{c}{ Botrytis sp. vs Trichoderma spp. } & $\begin{array}{c}\text { Crecimiento radial de } \\
\text { micelio (cm), } \mathbf{3} \text { dds. }\end{array}$ & $\begin{array}{c}\text { Prueba de Duncan } \\
(\boldsymbol{\alpha}=\mathbf{0 . 0 5})\end{array}$ \\
\hline Botrytis sp. vs T. atroviride (MHSJ01) & 2.41 & $\mathrm{a}$ \\
Botrytis sp. vs T. longibrachiatum (MJHU01) & 3.09 & $\mathrm{~b}$ \\
Botrytis sp. vs T. atroviride (MOBSHO03) & 3.17 & $\mathrm{bc}$ \\
Botrytis sp. vs T. strictipile (MCHT04) & 3.22 & $\mathrm{c}$ \\
Botrytis sp. vs T. harzianum (MHMLL04) & 3.39 & $\mathrm{~d}$ \\
Botrytis sp. vs T. atroviride (MHMLL06) & 3.39 & $\mathrm{~d}$ \\
\hline Botrytis sp. vs T. inhamatum (MOBSHO01) & 3.41 & $\mathrm{~d}$ \\
Botrytis sp. vs T. koningii (MHSJ1) & 3.43 & $\mathrm{~d}$ \\
\hline Botrytis sp. vs T. strictipile (MCHPS03) & 3.44 & $\mathrm{~d}$ \\
Botrytis sp. vs T. citrinoviride (MHMLL03) & 3.45 & $\mathrm{~d}$ \\
\hline Botrytis sp. vs T. koningii (MCHT11) & 3.46 & $\mathrm{~d}$ \\
Botrytis sp. vs T. harzianum (MOSA05) & 3.49 & $\mathrm{~d}$ \\
Botrytis sp. vs T. harzianum (MCHT07) & 3.69 & $\mathrm{e}$ \\
\hline
\end{tabular}

3.3. Competencia por espacio y nutrientes de Trichoderma spp. Frente a Botrytis sp. y testigos

Producto del análisis de varianza se encontró diferencias estadísticas altamente significativas $(p<0.01)$ en los promedios de áreas de los tratamientos estudiados para la competencia por espacio y nutrientes de Trichoderma spp. frente a Botrytis sp. y testigos, con un coeficiente de variación de $12,13 \%$.

La prueba de Duncan $(\alpha=0.05$ de probabilidad) mostro que la mayor área de medio ocupado fue 
por T. koningii (MHSJ11) con $24.58 \mathrm{~cm}^{2}$; seguido de T. koningii (MHSJ11) - Botrytis sp. con 23.81 $\mathrm{cm}^{2}$, T. inhamatum (MOBSHO01) - Botrytis sp. con $23.44 \mathrm{~cm}^{2}, T$. inhamatum (MOBSHO01) con $23.17 \mathrm{~cm}^{2}$, T. harzianum (MHMLL04) con 22,58 $\mathrm{cm}^{2}$ y T. atroviride MHSJ01 vs Botrytis sp. con $21,41 \mathrm{~cm}^{2}$ con diferencias significativas entre sí, probablemente Trichoderma tenga un mecanismos de rápida detección de hifas exploratorias y de reconocimiento de exudados del patógeno. Por otro lado, la cepa $T$. longibrachiatum (MJHU01) con $2.49 \mathrm{~cm}^{2}$ y $T$. longibrachiatum (MJHU01) - Botrytis sp. con 1.98 $\mathrm{cm}^{2}$ presentan una ocupación de espacio de desarrollo significativamente inferior al resto de los tratamientos; posiblemente se deba a que esta cepa sea muy exigente a condiciones de desarrollo in vitro o no sea muy competitiva frente a otro hongo.

La evaluación del área de desarrollo para cada cepa como testigo y enfrentado con Botrytis no presento similitud a nivel estadístico, siendo las cepas: T. harzianum (MHMLL04) con $22.58 \mathrm{~cm}^{2}$ y T. harzianum (MHMLL04) - Botrytis sp. con $21.14 \mathrm{~cm}^{2}$, T. atroviride (MHSJ01) - Botrytis sp. con $21.41 \mathrm{~cm}^{2}$, T. atroviride (MHSJ01) con 20.84 $\mathrm{cm}^{2}$, T. citrinoviride (MHMLL03) con $19.5 \mathrm{~cm}^{2}, \mathrm{~T}$. citrinoviride (MHMLL03) - Botrytis sp. con 15.69 $\mathrm{cm}^{2}$, T. atroviride (MHMLL06) con $18.48 \mathrm{~cm}^{2}, T$. atroviride (MHMLL06) - Botrytis sp. con 16.05 $\mathrm{cm}^{2}$, T. harzianum (MCHT07) con $17.79 \mathrm{~cm}^{2}$ y T. harzianum (MCHT07) - Botrytis sp. con 17.07 $\mathrm{cm}^{2}$, T. inhamatum (MOBSHO03) con $16.15 \mathrm{~cm}^{2}$ y T. inhamatum (MOBSHO03) - Botrytis sp. con $12.33 \mathrm{~cm}^{2}$ respectivamente. Excepto para $T$. longibrachiatum (MJHU01) como testigo y en enfrentamiento. Quizá podría deberse a características genéticas o por falta de competitividad frente a otro hongo. La superioridad de competición por espacio $\mathrm{y}$ nutrientes de las cepas de Trichoderma spp. sea condicionado por la tasa de crecimiento, seguido se presentan las mayores tasas de crecimiento para las cepas de Trichoderma: $T$. koningii (MHSJ11) testigo con $1.49 \mathrm{~cm} /$ día; $T$. koningii (MHSJ11) enfrentado con $1.46 \mathrm{~cm} /$ día; T. inhamatum (MOBSHO01) enfrentado con 1.42 $\mathrm{cm} /$ día; T. inhamatum (MOBSHO01) testigo con $1.48 \mathrm{~cm} /$ día, T. harzianum (MHMLL04) testigo con $1.42 \mathrm{~cm} /$ día y $T$. atroviride (MHSJ01) enfrentado con $1.38 \mathrm{~cm} /$ día. Para los resultados anteriores, Ahmad y Baker (1987) mencionan que la competencia por espacio y nutrientes es una característica del controlador biológico favorecida por la velocidad de crecimiento.

\subsection{Micoparasitismo}

En la escala de evaluación de micoparasitismo (mecanismo de acción antagónica) alcanzado por las cepas de Trichoderma al enfrentarse a Botrytis sp. las cepas: T. koningii (MHSJ11), T. atroviride (MHSJ01), T. atroviride (MOBSHO03), T. koningii (MCHT11), T. atroviride (MHMLL06), T. citrinoviride (MHMLL03), T. inhamatum (MOBSHO01) y T. harzianum (MCHT07) lograron alcanzar la clase 1 de la escala propuesta por Bell et al. (1982), por crecer completamente sobre la colonia de la cepa patógeno Botrytis sp. (MANB01) esto redujo el área de desarrollo y la formación de conidios de Botrytis; posiblemente, las cepas de Trichoderma tienen una mayor capacidad de detección quimiotrópica del patógeno a ello se suma la velocidad de su crecimiento de micelio, sobre el micelio del patógeno Botrytis sp.; en un segundo nivel se tiene a las cepas T. harzianum (MHMLL04), T. harzianum (MOSA05), T. strictipile (MCHPS03), T. strictipile (MCHT04) y T. longibrachiatum (MJHU01) los cuales alcanzaron una clase 2: logrando invadir dos 
terceras partes de la superficie del medio de cultivo, posiblemente a un menor grado de reconocimiento químico y físico del patógeno Botrytis (figura 2).

Los resultados de micoparasitismo, que incluyen como mecanismo de acción al crecimiento de micelio de las cepas Trichoderma spp. sobre el micelio de Botrytis sp., coinciden con los obtenidos por Elad et al. (1983), quienes evaluaron el micoparasitismo mediante la técnica de cultivos duales (patógeno antagonista) para las cepas de T. harzianum y $T$. hamatum con Rhizoctonia solani y Sclerotium rolfsii; determinando el crecimiento del biocontrolador (Trichoderma) sobre el hongo fitopatógeno ( $R$. solani o S. rolfsii). En efecto, el sobrecrecimiento del micelio de Trichoderma (clase 1 de la escala micoparasítica de Bell et al. 1982), es un carácter ventajoso en la disputa de colonizar un área (competición por espacio y nutrientes); esta es una manera de ejercer biocontrol, al reducir o detener completamente el desarrollo del micelio de un patógeno (Denis y Webster, 1971). Consecuentemente, Harman et al. (1981) sugieren que, aunque el micoparasitismo es el principal mecanismo de biocontrol para Trichoderma spp., existen otros como: adhesión y enrollamiento, competencia por nutrientes, reconocimiento, crecimiento quimiotrófico, la antibiosis, producción de antibióticos volátiles.

En la caracterización microscópica del enfrentamiento de cepas de Trichoderma frente Bortrytis, se observó que cuatro cepas de Trichoderma [T. koningii (MHSJ11), T. atroviride (MHSJ01), T. atroviride (MOBSHO03) y $T$. koningii (MCHT11)] no presentaron interacción hifal de enrollamiento; probablemente estas cepas cuenten con otro otros tipos de acción antagónica como segregación de sustancias quimicas como mencionan McAllister et al. (1994), quienes estudiaron la relación antagónica Trichoderma - Glomus mossae, observaron que, las hifas del biocontrolador en contacto con las del hongo Glomus no las enrollaron, afirmando que el micoparasitismo incluye segregación de antibióticos péptidos. Señalar que en una interacción hifal de enrollamiento posiblemente se dé segregación de sustancias antibióticas y/o enzimáticas. Los estudios realizados por Ochoa (2002) demostraron que las especies de Trichoderma spp. atacan a las hifas del hospedero por enrollamiento: enganchando o aprisionando las estructuras y penetrando la pared celular del hospedero, por secreción de enzimas líticas como proteinasas básicas, $\beta$-1,3-glucanasas y quitinasas; en efecto, la actividad antifúngica de las enzimas quitinolíticas tienen un papel importante en la lisis de la pared celular de los hongos fitopatógenos.

A nivel microscópico ocho cepas de Trichoderma [T. atroviride (MHMLL06), $T$. citrinoviride (MHMLL03), T. harzianum (MOSA05), T. inhamatum (MOBSHO01), T. harzianum (MCHT07), T. strictipile (MCHPS03), T. strictipile (MCHT04) y T. longibrachitum (MJHU01)] presentaron interacción hifal de enrollamiento. Estos resultados fueron similares a los reportados por Elad et al. (2000), quienes observaron ante un microscopio electrónico, enrollamiento de Trichoderma spp. en hifas de varios fitopatógenos, entre ellos Rhizoctonia solani, Sclerotium rolfsii, Fusarium oxysporum f. sp. radicis-lycopersici, Colletotrichum gloeosporioides y B. cinérea entre otros. Consecuentemente ocho cepas [T. atroviride (MHSJ01), T. atroviride (MOBSHO03), T. koningii (MCHT11), T. harzianum (MHMLL04), T. atroviride (MHMLL06), T. citrinoviride 
(MHMLL03), T. harzianum (MOSA05), T. inhamatum (MOBSHO01), T. harzianum (MCHT07) y T. strictipile (MCHPS03)] presentaron penetración en la hifa de Botrytis. Asimismo, Henis et al. (1983), al describir la acción de Trichoderma spp. sobre esclerocios de
Sclerotium rolfssi, resaltan que la capacidad de penetración de las hifas de Trichoderma, esta capacidad anidada un conjunto de mecanismos proprio de cada cepa mejora la eficiencia como un biocontrolador.
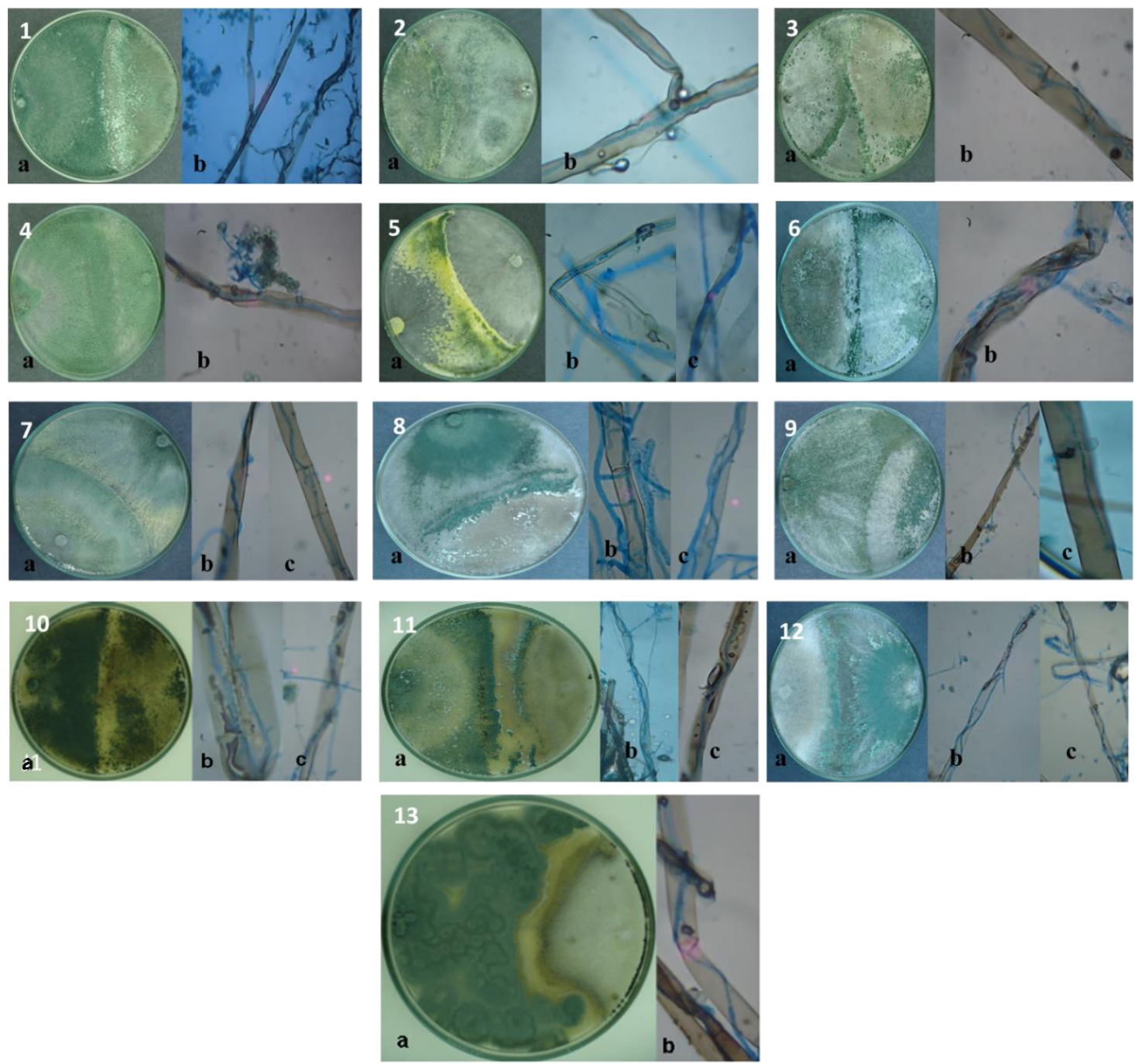

Figura 2. Comparativa de la capacidad antagónica por competición de sustrato (medio de cultivo) de Trichoderma spp. frente a Botrytis sp. a los 7 dds. 1. Enfrentamiento de T. koningii (MHSJ11) - Botrytis sp.: 1a. Enfrentamiento en medio PDA, 1b. Actividad lítica 40X; 2. Enfrentamiento de T. atroviride (MHSJ01) - Botrytis sp.: 2a. Enfrentamiento en medio PDA, 2b. Penetración de hifas 100X; 3. Enfrentamiento de T. atroviride (MOBSHO03) - Botrytis sp.: 3a. Enfrentamiento en medio PDA, 3b. Penetración de hifas 100X; 4. Enfrentamiento de T. koningii (MCHT11) - Botrytis sp.: 4a. Enfrentamiento en medio PDA, 4b. Penetración de hifas 100X; 5. Enfrentamiento de T. harzianum (MHMLL04) - Botrytis sp.: 5a. Enfrentamiento en medio PDA, 5b. Penetración de hifas 100X; 6. Enfrentamiento de T. atroviride (MHMLL06) - Botrytis sp.: 6a. Enfrentamiento en medio PDA, 6b. Penetración y enrollamiento de hifas 100X; 7. Enfrentamiento de T. citrinoviride (MHMLL03) - Botrytis sp.: 7a. Enfrentamiento en medio PDA, 7b. Enrollamiento de hifas 100X, 7c. Penetración de hifas 100X; 8. Enfrentamiento de T. harzianum (MOSA05) - Botrytis sp.: 8a. Enfrentamiento en medio PDA, 8b. Penetración de hifas 100X, 8c. Enrollamiento de hifas 100X; 9. Enfrentamiento de T. inhamatum (MOBSHO01) - Botrytis sp.: 9a. Enfrentamiento en medio PDA, 9b. Enrollamiento de hifas 40X, 9c. Penetración de hifas 100X; 10. Enfrentamiento de T. harzianum (MCHT07) - Botrytis sp.: 10a. Enfrentamiento en medio PDA, 10b. Penetración de hifas 100X, 10c. Enrollamiento de hifas $100 \mathrm{X} ; 11$. Enfrentamiento de T. strictipile (MCHPS03) - Botrytis sp.: 11a. Enfrentamiento en medio PDA, 11b. Enrollamiento de hifas 40X, 11c. Penetración de hifas 100X; 12. Enfrentamiento de T. strictipile (MCHT04) - Botrytis sp.: 12a. Enfrentamiento en medio PDA, 12b. Enrollamiento de hifas 100X, 12c. Penetración de hifas 100X y 13. Enfrentamiento de T. longibrachitum (MJHU01) - Botrytis sp.: 13a. Enfrentamiento en medio PDA, 13b. Enrollamiento de hifas 100X. 


\subsection{Grado de inhibición}

Los resultados del análisis de varianza para el grado de inhibición que tuvieron las cepas de Trichoderma spp. frente a Botrytis, mostro diferencias estadísticas altamente significativas ( $p<0.01)$, con un coeficiente de variabilidad de $6.39 \%$.

La comparación de medias por Duncan $(\alpha=0.05$ de probabilidad) indica un mayor grado de inhibición $(39,3 \%)$ para Botrytis sp. frente a $T$. atroviride (MHSJ01); destacando a T. atroviride (MHSJ01) y su posible por su activación rápida de mecanismos de acción como: competencia (rápido desarrollo sobre el medio), micoparasitismo y antibiosis (segregación de antibióticos y expulsión de antibióticos volátiles) propio de esta cepa, en respuesta al reconocimiento del patógeno Botrytis. En segundo lugar, los enfrentamientos Botrytis sp. - T. longibrachiatum (MJHU01) con $22.01 \%$ y Botrytis sp. - T. atroviride (MOBSHO03) con 20.04 $\%$. Por el contrario, el enfrentamiento de Botrytis sp. - T. harzianum (MCHT07) con $6.81 \%$ presenta un grado de inhibición estadísticamente inferior al resto; posiblemente sus mecanismos como competencia, micoparasitismo y antibiosis no sean eficientes. Cabe agregar, que cepas de Trichoderma identificadas como una misma especie no presentan necesariamente un efecto de inhibición estadísticamente igual, como es el caso de: Botrytis sp. - T. atroviride (MOBSHO03) con $20.04 \%$ y Botrytis sp. - T. atroviride (MHMLL06) con $14.57 \%$ y finalizó Botrytis sp. T. strictipile (MCHT04) con $18.79 \%$ y Botrytis sp. - T. strictipile (MCHPS03) con $13.25 \%$. También Suarez et al. (2008), experimentaron con aislamientos nativos y comerciales de $T$. harzianum, sobresaliendo aislamiento nativo
(TCN-014) y uno comercial (TCC-005), ambos resultaron tener mayores habilidades de micoparasitismo (grado 4 y completo), competencia por espacio y nutrientes $(7,33 \mathrm{~cm}$ y $7,32 \mathrm{~cm}$ de radio de crecimiento a $10 \mathrm{dds}$ ) e inhibición del crecimiento de micelio $(65.32 \%$ y $70.6 \%$ ) de los aislamientos de Fusarium solani a nivel in vitro.

\section{CONCLUSIONES}

Se encontró diversos niveles de antagonismo de las cepas de Trichoderma spp. frente a Botrytis sp. para las variables: en el crecimiento radial de micelio de Trichoderma spp. por efecto del enfrentamiento contra Botrytis sp. y sus testigos, las cepas: T. koningii (MHSJ11) testigo y enfrentado; T. inhamatum (MOBSHO01) testigo y enfrentado y T. harzianum (MHMLL04) testigo fueron superiores al resto. Asimismo, la mayor contención del desarrollo de micelio de Botrytis sp. por efecto del enfrentamiento contra Trichoderma spp. lo hizo la cepa T. atroviride (MHSJ01). En la competencia por espacio y nutrientes por efecto del enfrentamiento Trichoderma spp. frente a Botrytis sp. y sus testigos, sobresalió la cepa T. koningii (MHSJ11) con $24.58 \mathrm{~cm}^{2}$ a los 3 dds. En micoparasitismo, las cepas que lograron la escala 1 fueron: $T$. koningii (MHSJ11), T. atroviride (MHSJ01), T. atroviride (MOBSHO03), T. koningii (MCHT11), T. atroviride (MHMLL06), T. citrinoviride (MHMLL03), T. inhamatum (MOBSHO01) y $T$. harzianum (MCHT07) y con un mayor grado de inhibición (39,3\%) para T. atroviride (MHSJ01).

\section{Referencias}

Ahmad, J. \& Baker, R. (1987). Rhizosphere competence of Trichoderma harzianum. Phytopatology. 77: 182-189. 
Barnett, H. \& Hunter, B. (1998). Illustrated genera of imperfect fungi. Ebook. Minnesota, Estados Unidos. Cuarta adición. 218p.

Bell, D., Well, H. \& Markham, C. (1982). In vitro antagonism of Trichoderma species against six fungal plant pathogens. Phytopathology 72: 379-382.

Campbell, R. (1989). El control biológico de patógenos microbianos de las plantas. Cambridge University Press. New York, USA. 218 p.

Chambers, S. \& Scott, E. (1995). In vitro antagonism of Phytophthora cinnamomi an $P$. citrícola by isolates of Trichoderma spp. and Gliocladium virens. Journal of Phytopathology. 471-477 p.

Dennis, L. \& Webster, J. (1971). Antagonistic properties of species groups of Trichoderma. III Hyphal interaction. 369p.

Elad, Y. (2000). Biological control of foliar pathogens by means of Trichoderma harzianum and potential modes of actions. Biocontrol Science and Technology. 499 p.

Elad, Y., Chet, I., Boyle, P. \& Henis, Y. (1983). Parasitism of Trichoderma spp. on Rhizoctonia solani and Sclerotium rolfsii - Scanning electron microscopy and fluorescence microscopy. Phytopathology 85-88 p.

Esparza, L. (2009). Efectividad in vitro de cepas nativas de Trichoderma spp. en aislados de Phytophthora parasitica D. obtenidos en plantas de Jamaica. Tesis. Colegio de Postgraduados. Montecillo, México. 69 p.

Harman, G., Chet, I. \& Baker, R. (1981). Factors affecting Trichoderma hamatum applied to seed as a biocontrol agent. Phytophatoloy. 569-672 p.

Henis, Y., Adams P., Lewis, J. \& Papavizas, G. (1983). Penetration of sclerotia of Sclerotium rolfsii by Trichoderma spp. Phytopathology 143 - 146 p.
Infante, D., Martínez, B., Gonzales, M. y Reyes, Y. (2009). Mecanismos de acción de Trichoderma frente a hongos fitopatógenos. Revista de protección vegetal. Univers. Agraria de La Habana. La Habana, Cuba. 11 p.

McAllister, C., García, I., Godeas, A. \& Ocampo, J. (1994). Interactions between Trichoderma koningii, Fusarium solani and Glomus mosseae: Effects on plant growth, arbuscular mycorhizas and the saprophyte inoculants. Soil Biology and Biochemistry. 260 p.

Memenza, Z. (2009). Control biológico in vitro de Botrytis cinerea (Pers) mediante el uso de hongos antagonistas, en vid (Vitis vinifera). Tesis. Lima, Perú. Universidad Nacional Mayor de San Marcos. 75 p.

Ochoa, M. (2002). Antibiosis y micoparasitismo de cepas nativas de Trichoderma spp. (Hyphomicetas: Hyphales), sobre Mycosphaerella fijiensis (Loculoascomycetes: Dothideales). Tesis de Maestría en el Área de Biotecnología. Univers. de Colima. México. 102 p.

Pitt, J. \& Hocking, A. (2009). Fungiand Food Spoilage. Ebook. Australia. Tercera edición. 524 p.

Rosales, B. (2012). Aislamiento, identificación y caracterización cultural de cepas nativas de Trichoderma spp. en la provincia de Oxapampa. Tesis. Universidad Nacional Daniel Alcides Carrión. Oxapampa, Perú. 147 p.

Sarro, B., Lara, M. \& Fernández, C. (2010). Evaluación in vitro de la capacidad antagonista de Trichoderma lignorum FEEP TL0601 frente a Fusarium oxysporum $\mathrm{f}$. sp. lycopersici. PHYTOMA España, 225: 47-50.

Suarez, M., Fernández, B., Osvaldo, V., Gámez, C. y Páez, R. (2008). Antagonismo in vitro de Trichoderma harzianum Rifai sobre Fusarium solani Mart. Sacc. asociado a la marchitez en maracuyá. Revista Colombiana de Biotecnología. Colombia. 9 p 